\title{
Development of the ALIS IP Ontology: Merging Legal and Technical Perspectives
}

\author{
Claudia Cevenini ${ }^{1}$, Giuseppe Contissa ${ }^{2}$, Migle Laukyte ${ }^{3}$, Régis Riveret ${ }^{4}$, and \\ Rossella Rubino ${ }^{5}$ \\ ${ }^{1}$ CIRSFID, University of Bologna, Italy, claudia.cevenini@unibo.it \\ ${ }^{2}$ CIRSFID, University of Bologna, Italy, giuseppe.contissa@unibo.it \\ ${ }^{3}$ CIRSFID, University of Bologna, Italy, migle.laukyte@unibo.it \\ ${ }^{4}$ CIRSFID, University of Bologna, Italy, regis.riveret@unibo.it \\ ${ }^{5}$ CIRSFID, University of Bologna, Italy, rossella.rubino@unibo.it
}

\begin{abstract}
The paper is partly based on research done for the EU-funded (IST2004-2.4.9) project ALIS (Automated Legal Intelligent System) on Intellectual Property Law. We describe the development process of the ALIS Intellectual Property ontology from both a legal and a technical perspective. We discuss the scope and the features of this IP ontology, the lessons learned, and the problems solved. This is done by comparing our ontology (the ALIS IP ontology) with the IPRonto ontology, which too is dedicated to IP. The paper also points out the benefits of both the ALIS system in general and the ALIS IP ontology in particular. Future development of ALIS will involve expanding its ontology to also include law and trademark law. Once these three legal ontologies are in place, they will be consolidated into a single ontology that will provide the framework for a general IP ontology.
\end{abstract}

Keywords: Intellectual property law, legal ontology, IPROnto, ALIS system

\section{Introduction}

It was as early as the end of the 20th century that computer scientists began to explore the possibility of using ontologies for the law [1, 2]. Yet, for all that experience, we cannot say that we have made much progress today: while the tools for building legal ontologies have definitely improved, law is usually conceptualized in the abstract, without entering into particular areas of law, such as labour law, insurance law, and intellectual property law. Today, there is only one ontology for intellectual property law (IP law): it is called IPROnto [3]. This was the ontology from which we took inspiration and with which we compared our own. In this paper we attempt to create another IP ontology, called the ALIS IP ontology, based on research done under the ALIS project. The paper is

Please use the following format when citing this chapter: 
organized as follows: Section 2 provides an overview of the ALIS project and the role an IP ontology plays in it. Section 3 discusses two perspectives that we adopted: the legal perspective (3.1) and the technical one (3.2). Section 4 presents the method used. Section 5 brings the ALIS IP ontology into comparison with IPROnto. Section 6 presents the lessons we learned and the problems we solved in the process of building our ontology. Section 7 presents our conclusions and an outlook for the future research.

\section{ALIS and Its Ontology}

ALIS - a project funded under the 6th Framework Program-stands for Automated Legal Intelligent System (IST-2004-2.4.9). The project is aimed at modeling a legal system for IP law and providing citizens and public as well as private organizations with fast and reliable access to the European IP law.

The problems the project is intended to solve are thus both legal and technical. There are four ways in which this is so. First, ALIS seeks to apply to IP law different kinds of research in artificial intelligence, ranging from knowledge representation and reasoning to game theory. Second, while most of the work involved in building such a system is in computer science, the people who will benefit from it will be in the first place will be the lawyers. Third, the legal problems connected with IP law are being solved using technological solutions, namely, the ALIS system. And fourth, IP law is at the same time an area of law designed to protect technological works of mind, providing for authors and creators a guarantee that their rights will be protected. (The ALIS system is no exception.)

The reason why we decided to make the ALIS system specific to IP law is that we believe IP rights to be a central concern in European society today, a concern owed to the progress of science and technology, and Internet technologies in particular. Indeed, there is a time lag between law and technology, with technology renewing on a monthly basis, or nearly so, and law taking years to catch up. The ALIS system will try to contribute toward bridging this gap.

The scientific community is working actively and organizing many conferences on legal ontologies and neighbouring technologies (witness LOAIT'05, LOAIT07, SW4Law07). These conferences have given birth to a number of publications aside from their proceedings, including Law and the Semantic Web: Legal Ontologies, Methodologies, Legal Information Retrieval, and Applications [4] and, recently, two special issues of the journal Artificial Intelligence and Law $[5,6]$.

The ALIS system requires at least two main ontologies: the IP ontology and the game-theory ontology. The IP ontology is useful for processing the legal documents crawled by ALIS system. This is done analyzing the contents of such documents and mapping these contents onto generic concepts. 
Practical use of the ALIS system (as enabled by this ontology) brings benefits to both the private and the public sector. The copyright collection societies (public sector) can apply this system in e-governance, while companies and enterprises in e-commerce (private sector) can take advantage of the ALIS system for DRM management, for systems by which to manage IP contracts automatically, and finally for agent-based systems dedicated to IP products. Further, the ALIS system is important in labour relations (in both the private and the public sector), where the authorship of a creation (mainly software products) may provoke disputes, which institutions and organizations prefer to prevent.

\section{Two Perspectives in Creating the ALIS IP Ontology}

Every ontology is an agreement, a social contract among people driven by a common purpose [7]. The ALIS legal ontology is likewise an agreement-an agreement between lawyers and software engineers who have to work together in this initiative. This section examines the ontology-building process from these two perspectives of the lawyer and the software engineer.

\subsection{The Legal Perspective}

For the legal perspective we tried to follow French IP law strictly construed, without legal interpretation, without taking into account the way in which other laws come to bear, and without changing the legal terminology: the terms and concepts used in the ALIS IP ontology are the same as those used in French IP law. This ontology thus follows the letter of the French law and so takes from it its normative shape: this was made necessary by the aims the ALIS project is meant to achieve, especially the aim of making laws easier to draft. ALIS includes an isomorphic [14] representation of norms through a formalized set of rules. The same approach - strict adherence to the body of norms in question - was taken for the ALIS IP ontology, even though this ontology was built to achieve not so much isomorphism as comprehensiveness and correctness [1].

The concepts making up the ontology were selected by including, from the start, all the copyright terms that could be relevant to the same ontology: these include the fundamental legal terms of IP law, such as author and work of mind; the different types of works of mind recognized under the law (collective, collaborative, single, and composite); and other terms such as moral right and economic right.

This selection of concepts did, however, pose a few problems. One of these was list of works of mind. The law describes this as the list of all the works of mind protected by copyright, but it does not also define a work of mind, and so it leaves open the possibility of any further number of works making it into the list, 
so long as they enjoy copyright protection. This is therefore a cautious list: an incomplete list containing only such works as are encountered on a daily (or almost daily) basis, which means that it may take in combinations of such works, or works deriving from them, or any other outcome of human creativity. In other words, there is room for judicial interpretation on case-by-case basis.

But this way of framing the law and reasoning around it does not help toward building an ontology: from ontological point of view, whatever terms are not in the ontology do not exist in the law. The problem of works not mentioned in the list but which might enjoy copyright protection anyway might be solved in the future by updating the ontology with new concepts as necessary, on case-by-case basis. Otherwise the ontology could fall behind what the law deems a work of mind.

Every concept the lawyers chose to bring into the ontology was checked with software engineers to see whether the concept (a) falls under a rule and (b) is relevant within the overall conceptual map of copyright law. For instance, terms such as contract for hire and service contract do come under a rule-this being the rule that no contract made by the author of a work of mind can undermine the author's ability to enjoy his or her rights to that work-but they are nonetheless irrelevant to IP law, and for this reason we decided not to include them into the ALIS IP ontology.

In making a selection of terms for the ontology, we drew up a glossary explaining what each of these terms means. The meanings so ascribed in the glossary is broader than that which these terms have under French IP law, and this was done on purpose, the point being to include all the possible meanings of certain term that the user might be interested in.

\subsection{The Technical Perspective}

The technical perspective involves the three concepts of author, work of mind, and intellectual-property rights, a triplet of fundamental importance to French IP law. In what follows, we will describe these concepts separately and list related concepts in italics.

The first concept in the ALIS IP ontology is that of work of mind, which is a creative expression of intellectual work and may be of different types. French IP law defines the following types of works of mind: abstract authorship, address, applied art work, architectural work, artistic writing, audiovisual work, book, choreographic work, cinematographic work, circus act, collection of miscellaneous works or data, dramatic musical work, dramatic work, drawing work, dumb-show work, engraving work, fashion work, feat, geographical map work, graphical work, illustration work, lecture, literary writing, lithography work, musical composition with words, musical composition without words, painting work, pamphlet, photographic work, photography analogous work, plan 
work, pleading, scientific writing, sculpture work, sermon, single work, sketch work, software work, three dimensional work, typographical work. ${ }^{1}$

Furthermore, this list does not specify works of mind exhaustively, for these also get classified on the basis of the manner in which they are created, as collective, composite, or single works, or again as works executed in collaboration.

A work of mind is further defined by a term of protection, specifying the period during which there is an exclusive right to exploit the same work.

The second concept of the triplet is that of author. A work of mind is created by an author, who may be a natural or legal person, and who may also be anonymous or pseudonymous. Hence, a work of mind may itself be anonymous or pseudonymous.

The third main concept in the ontology is that of intellectual property rights, which every author is entitled to, and which may also be referred to as incorporeal property rights. French IP law distinguishes between moral and economic rights. Moral rights protect an author's personality and reputation. Economic rights enable the copyright owner to benefit economically from the work's exploitation, and they may be transferred to an economic right holder (publisher, editor, producer) either in part or in full.

French IP Law identifies nine types of moral rights as follows: right of disclose under the name, right of disclose under to direction, right of divulge work, right of make a collection, right of reconsider assignment of exploitation, right of respect for authorship, right of respect for name, right of respect for work, right of withdraw assignment of exploitation.

Economic rights are in turn divided in two groups of rights: right of performance and right of reproduction. The former is the right to communicate the work to the public by any process whatsoever (perform dramas or lyrics, present or project or recite publicly, telediffuse or transmit also through satellite). The latter is the right to fix a work into a physical medium by any process enabling it to be indirectly communicated to the public (publication, cast, draw, engrave, execute, make photos, print, use graphic or mechanical process).

All italicized concepts in this section are represented as OWL classes connected by datatype or object properties. Fig. 1 is a UML diagram showing the relationships between the main concepts of French IP law.

\footnotetext{
${ }^{1}$ The terms in this list, as well as in all further lists, appear in alphabetical order and are lifted as such from the English translation of the French IP law: for this reason, we have chosen not to change them even when they are nonstandard or seem to make little sense. The same applies to all italicized terms and expressions from here on out.
} 


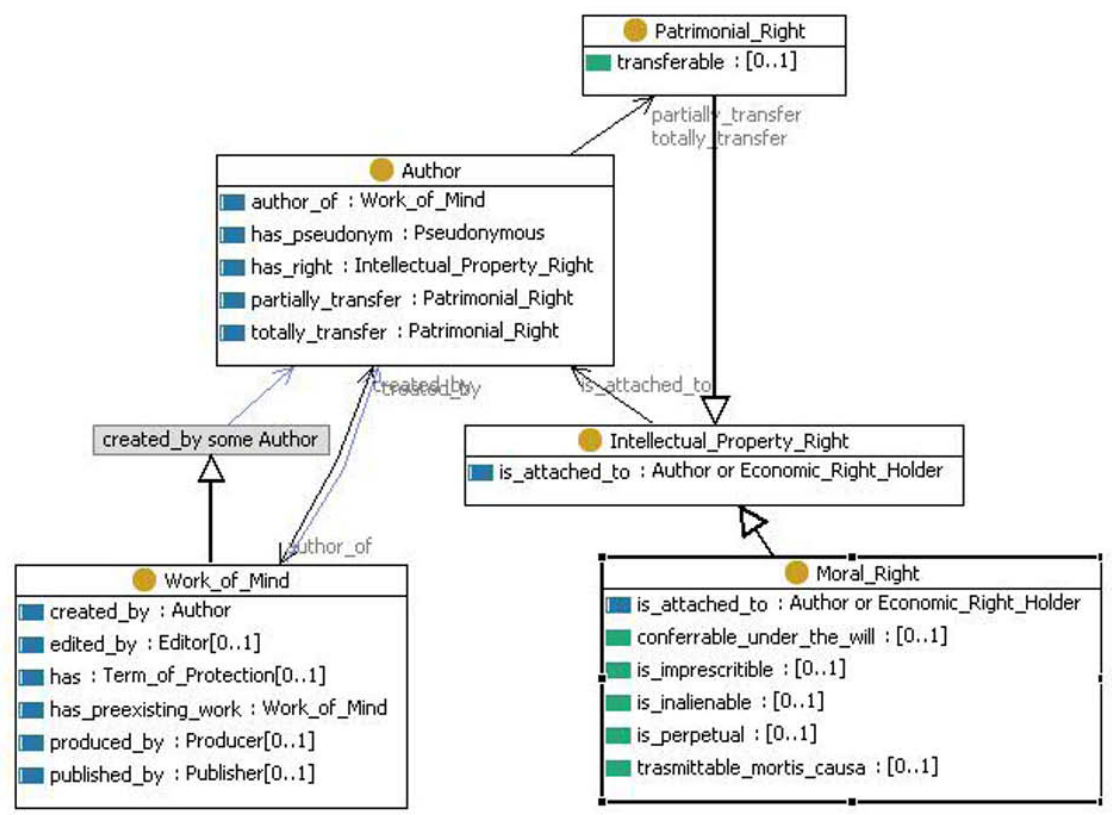

Figure 1 UML diagram laying out main IP concepts

The ALIS consortium, taking inspiration from IPROnto, created the model to illustrate the lifecycle of the ALIS IP ontology. This is called the OCATU Model [13].

The lifecycle of an IP case can be defined by the five stages making up the global process of the OCATU Model:

1) organize - team setup and creation preparation

2) create - source transformation

3) assert - assert the created copyrights (works of mind)

4) transfer - transfer rights

5) use - use the created copyrights (works of mind)

By combining all key data through different stages, we get a tree structure representing the key facts in a uniform language. Case after case, the tree's branches are enriched and yield a common database of copyright cases. Any new case can then be placed in the tree in the form of a unique path presenting key events.

When a particular IPR case is analyzed, not all the stages appear explicitly, but all are necessary to understand the case. The OCATU Model is designed to structure real cases, especially copyright cases, so as to facilitate the development of a game theory ontology. 
The process starts from the creation of an object that may be subject to copyright and ends with the object's use. It forms a sequence in which no stage can begin until the previous one has been when completed. The final stage ("use") may generate another creation, in which case the five-stage process can be represented as a cycle.

If we want to generate a path from the "organize" stage to the "use" stage, we have to design a "solution tree," which includes all the actions a user may take or does take. Three kinds of paths can be distinguished depending on the point of view one takes: action paths, showing all the actions a user has taken; status paths, showing the entire status resulting from user's actions; and complete paths, which combine the two other paths.

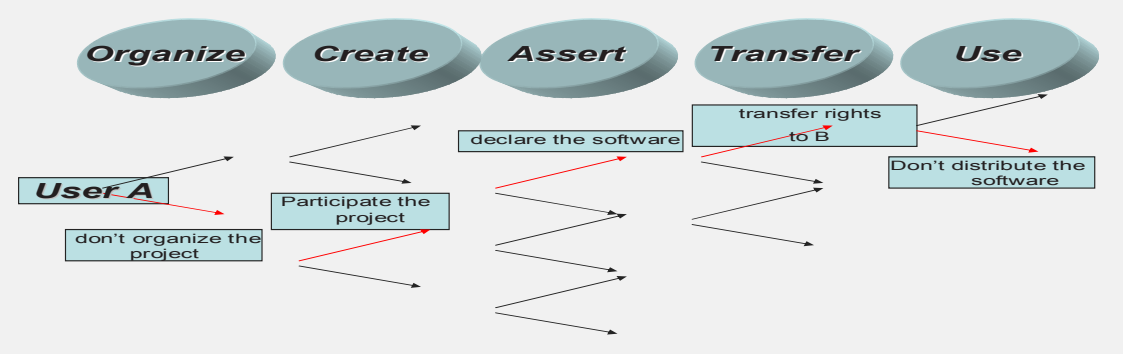

Figure 2 Status path of OCATU model

\section{Method}

In designing the ALIS IP ontology, we followed the main stages described in [10], which consist in determining the scope of the ontology; considering the reuse of its concepts; listing these terms; defining a taxonomy, properties, facets, and instances; and checking for anomalies. Moreover, we used the text of the French IP law to extract rules and model them in Prolog, and we also extracted definitions and formalized them in the OWL language.

Like any development process, so the process of developing an ontology is not linear: some of its stages will have to be iterated, and at any point it may be necessary to backtrack. Thus, for example, we have extended the ontology with new concepts from the legal rules expressed in the Prolog language.

The tool chosen for creating the ALIS ontology is Protegé. But we also used TopBrain Composer, which produces a very useful UML-like documentation but is not free.

The two ontologies developed for ALIS are used in the ALIS knowledge-base platform to support different tasks, including document annotation, game-theory reasoning, checking for legal compliance, and transcribing law into RuleML rules. 
Completing these tasks makes it necessary to access the different concepts and properties defined in the ALIS ontologies, and it also requires tools that can reason about such concepts and properties for a number of purposes, such as to detect contradictions or infer new properties. The ALIS consortium decided to develop an ontology engine using the Pellet reasoner [12].

\section{IPROnto and Comparison with the ALIS IP Ontology}

This section describes the IPR Onto ontology, which we took as a benchmark in building ours, and which to date remains the de facto benchmark for all ontologies in IP law. The table below (Table 1) compares the ALIS IP ontology with IPROnto.

IPROnto's upper ontology, selected to provide a robust ontology basis for interoperability, is SUMO, a result of the SUO IEEE WG [8] attempt to establish a standard upper ontology.

Furthermore, IPROnto uses the World Intellectual Property Organization (WIPO) [9], which is defining a common legal framework for IPR, to complete the legal parts of the ontology.

Following are the main legal concepts we analyzed: agreement, which takes two dependent elements, namely, IPRContract and IPRLicense; IntellectualPropertyRight, associated with multimedia digital content for automatic e-commerce on the Internet (it includes copyrights, sui generis rights (applicable to databases) and neighbouring rights (which apply in particular to singers and performing artists); copyrights, which include moral rights and exploitation rights (or economic rights); and LegalEntity, which divides into two categories, namely, CorporateLegalEntity and NaturalLegalEntity. A work protected by copyright has a lifecycle that establishes a flow among the several IPROnto legal concepts. The lifecycle involves roles (Creator, RightsHolder, ContentProvider, MediaDistributor) as well as actions (create, transfer, transform).

We have chosen to not use IPROnto for two main reasons. The first one is that the aim of IPROnto is totally different from ALIS IPR Ontology. Indeed, while IPROnto Onto aims at facilitating the automation and interoperability of IPR frameworks, ALIS Ontology is devoted to ensure that the templates, which will serve as users interface, were consistent with the information required by the ALIS system.

The second reason is that the ALIS project limited the scope of its system to copyright and the ALIS consortium chosen, as reference, the French IPR Law. This was due to the fact that the system validation will be performed by a French partner. We have therefore analyzed just the legal concepts cited in this text. 
Table 1 The ALIS IP Ontology and IPROnto compared

\begin{tabular}{|l|l|l|}
\hline Area & ALIS IPR Ontology & IPROnto \\
\hline Purpose & $\begin{array}{l}\text { 1) to better manage legal documents repositories; } \\
\text { 2) to make the document-retrieval system more } \\
\text { versatile; } \\
\text { 3) to create a common layer between legal and } \\
\text { game-theory concepts; } \\
\text { 4) to create a general "shared" language enabling } \\
\text { the modules and the Web services of the ALIS } \\
\text { architecture to exchange information; thus, for } \\
\text { example, the GUI module will be "ontology- } \\
\text { based," connecting every user input to a particular } \\
\text { norm; this norm might establish a certain } \\
\text { obligation for the user, who in game theory also } \\
\text { figures as a player, whose strategy will } \\
\text { consequently take that obligation into account. }\end{array}$ & $\begin{array}{l}\text { To facilitate the automation } \\
\text { and interoperability of IPR } \\
\text { frameworks, integrating } \\
\text { both the Rights Expression } \\
\text { Language and the Rights } \\
\text { Data Dictionary. }\end{array}$ \\
\hline $\begin{array}{l}\text { Upper } \\
\text { Ontology }\end{array}$ & $\begin{array}{l}\text { LKIF-core, which embeds the concepts of } \\
\text { Manifestation and Expression. }\end{array}$ & $\begin{array}{l}\text { SUMO, which does not } \\
\text { embed the concepts of } \\
\text { Manifestation and }\end{array}$ \\
& $\begin{array}{l}\text { Expression but rather } \\
\text { describes them through a } \\
\text { new, specifically designed } \\
\text { sub-ontology. }\end{array}$ \\
\hline WIPO? & No & Yes \\
\hline Life Cycle & Yes (OCATU Model) & Yes \\
\hline Granularity & Yes & No \\
\hline
\end{tabular}

\section{Lessons Learned and Problems Solved}

First thing we learned is that the larger the domain an ontology seeks to cover, the more the ontology will be abstract. Abstractness is precisely the feature of IPROnto, whose domain is much larger than that covered by the ALIS IP ontology, which confines itself to a specific part of French IP law and describes it with highly accurate terms and concepts.

The abstractness of IPROnto may be owed to its direct use of two abstract legal sources, namely, the Berne Convention and the WIPO Copyright Treaty, both of which are sketched out in broad strokes and serve mainly as guidelines for the domestic law of other countries. The ALIS IP ontology also draws on these two sources but does so indirectly, meaning that the ontology's direct source is the French IP law, which is modelled on the WIPO IP sources and so (like any other national IP law) acts to concretize the WIPO ideas and guidelines. 
The use of French IP law is also to account for other features that distinguish the ALIS IP ontology from IPRonto.

First, certain concepts mean the same but are expressed using different terms: IPRonto has exploitation rights, corresponding to patrimonial rights in the ALIS IP ontology. Another example is creator (IPROnto) and author (ALIS IP ontology). The division of economic rights is also different: while IPRonto subdivides exploitation rights into transformation rights, communication rights, distribution rights, and reproduction rights, the ALIS IP ontology divides patrimonial rights into only two classes, that is, rights of performance and rights of reproduction.

Second, because the ALIS IP ontology has a more restricted domain, it does not yet have the concepts or the French equivalents of SuiGenerisRights, NeighbouringRights, WithdrawalRight, ContentProvider, and MediaDistributors. These concepts can be found only in IPRonto.

Third, even though the two ontologies share the concept of Moral Right, the ALIS IP ontology specifies it in greater detail, thus enables users (especially nonlawyers) to have a better grasp of its meaning even without looking up its definition.

Fourth, the ALIS IP ontology makes it is possible to specify which economic rights transfer to the right-holder and which remain with the author(s): this is especially evident in comparison with IPROnto, where such specification is impossible.

Fifth, the ALIS IP ontology specifies the concept of Right Holder through the constituent concepts of Publisher, Editor, Producer, and Performer.

And sixth, the ALIS IP ontology specifies the concept of Work of Mind using the list of works that the French IP law deems amenable to copyright protection in France. Also, works of mind are further specified by classifying them on the basis of the manner in which they are created, as collective, composite, or single work, or as works created in collaboration.

Another lesson we have learned is that focusing on a well-defined domain makes it possible to exclude terms from other domains, thus avoiding what happens in IPROnto, which takes in terms foreign to the IP domain, such as Customer. This concept was introduced into IPROnto because this ontology covers not only IP law but also addresses the domain of commerce. Embracing two domains will enlarge the ontology's field of application, to be sure, but it also makes it more difficult to understand and manage this ontology, especially if designed (at least on paper) to cover only one of the two fields of application from which it draws its concepts.

We also realized how much work is still left to be done: the ALIS IP ontology covers only the main chunk of the legislative part of French IP, and its concepts therefore not only fail to account for the whole of the legislative part but also leave out the regulatory part. This is the reason why the ontology is still without many concepts, such as that of agreement, that IPROnto does, by contrast, include. 
The general concepts of the ALIS IP ontology come from LKIF-core [11]. The lesson learned here is that it is important to share the tools of the other European projects we have taken part in. Further, the LKIF-core ontology makes it possible to connect the ALIS IP ontology with business-intelligence tools, such as KnowledgeTools and RuleBurst, and so these tools can use the ALIS IP ontology.

We can now analyze the problems we set out to solve. The first problem is that the ALIS IP ontology is based on the English version of French IP law: we worked with the English version of French concepts, and this made it necessary to go back to the original at every turn to figure out what the translation meant or simply to make sure it was correct.

This problem connects to another we faced, which is that the French IP law quite often uses different words for the same concepts. So here, too, we had to study the text closely at many places to determine whether we were dealing with a single concept designated by two synonyms (which was most often the case) or whether the two words in question designated two different concepts (which did happen on a few occasions). This acted as a reminder of the problems involved in legal drafting and how important it is to produce a good text.

The last problem was that of deciding, for some of the concepts involved, whether they are better represented as classes or as properties. For example the concept of collaborative work should be represented as a class or as a property of a work of mind? We chose to represent the four ways of creating work of mind as separate classes so that these classes, in consequence, could have their properties.

\section{Conclusions and Outlook}

We have explained why the ALIS project needs an IP ontology and how this ontology was built proceeding from both a legal and a technical perspective. We have also compared this ontology to the only other existing IP ontology, called IPROnto. Further, we discussed the method used, the lessons learned, and the problems faced, for which we sought a solution.

The method used to create the ALIS IP ontology can be implied as a model for the construction of other legal ontologies that may be of direct interest to commerce. This holds in particular to the legal ontologies for business law, insurance law, labour law, and contract law.

We hope that in this effort to build an ontology we have done a service to other scholars with an interest in these issues.

We will continue in this effort. Apart from enriching the ALIS IP ontology with all of the concepts in French IP law, we will be expanding the ALIS ontology to also include patent and trademark law. Once these three legal ontologies are in place, we will consolidate them into a unique ontology making up the framework for a general IP ontology. 


\section{References}

1. Valente A.: Legal knowledge engineering. A modeling approach. IOS Press, Amsterdam (1995).

2. Visser P.R.S, Bech-Capon T.J.M.: The formal specification of a legal ontology. In: Van Kralingen et al. (eds.), Proceedings of JURIX 96 (pp. 15-24). Tilburg University Press, Tilburg (1996).

3. Delgado J., Gallego I., Llorente S., Garcia R.: Regulatory ontologies: An intellectual property right approach. In: On the move to meaningful internet systems, OTM Workshop, pp. 621635, Springer, Berlin (2003).

4. Benjamins R., Casanovas P., Gangemi A., Breuker J. (eds.): Law and the semantic web: legal ontologies, methodologies, legal information retrieval, and applications. Lecture Notes in Computer Science, Springer, Berlin (2005).

5. Lehmann J., Biasiotti M.A., Francesconi E., Sagri M.T. (eds.): Legal knowledge extraction and searching \& legal ontology applications. Special issue of Artificial Intelligence and Law, n. 7 (2007).

6. Breuker J., Tiscornia D., Winkels R., Gangemi A. (eds.): Ontologies for law. Special issue of Artificial Intelligence and Law, n. 4, December (2004).

7. Gruber T.: Every ontology is a treaty (2004). Available via SIGSEMIS bulletin http://tomgruber.org/writing/sigsemis-2004.pdf. Accessed 7 March 2008.

8. Standard Upper Ontology (SUO) IEEE Working Group. Available via https://suo.ieee.org. Accessed 3 March 2008.

9. World Intellectual Property Organization. Available via https://www.wipo.org. Accessed 7 March 2008.

10. Noy N.F., McGuinness D.L.: Ontology development 101: A guide to creating your first ontology. Stanford knowledge systems laboratory technical report KSL-01-05 and Stanford medical informatics technical report SMI-2001-0880 (2001).

11.Breuker J., Hoekstra R., Boer A., van den Berg K., Rubino R., Sartor G., Palmirani M., Wyner A., Bench-Capon T.: OWL ontology of basic legal concepts (LKIF-Core). Deliverable 1.4, Estrella (2007). Available via http://www.estrellaproject.org/lkif-core/. Accessed 14 March 2008.

12. Pellet Reasoner. Available via http://pellet.owldl.com/. Accessed 14 March 2008.

13. ALIS Deliverable D4.2: Description of the framework. Available via https://www.myndsphere. com/gm/document-1.9.32640/ALIS_WP5_D5.1a.doc. Accessed 8 March 2008.

14. Bench-Capon T.J.M, Coenen F.P.: Isomorphism and legal knowledge based systems. Artificial Intelligence and Law 1(1), pp. 65-86 (1992). 